\title{
Aquaporins in Cerebrovascular Disease: A Target for Treatment of Brain Edema?
}

\author{
J. Badaut ${ }^{\mathrm{a}, \mathrm{b}}$ S. Ashwal ${ }^{\mathrm{a}} \quad$ A. Obenaus ${ }^{\mathrm{a}, \mathrm{c}-\mathrm{e}}$ \\ Departments of a Pediatrics, b Physiology, ${ }^{c}$ Radiology, and ${ }^{d}$ Radiation Medicine, Loma Linda University

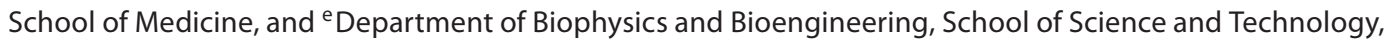 \\ Loma Linda, Calif., USA
}

\section{Key Words}

Stroke $\cdot$ Edema $\cdot$ Water channel $\cdot$ Neuroimaging $\cdot$ Apparent diffusion coefficient $\cdot$ Neurovascular unit $\cdot$ Blood-brain barrier

\begin{abstract}
In cerebrovascular disease, edema formation is frequently observed within the first 7 days and is characterized by molecular and cellular changes in the neurovascular unit. The presence of water channels, aquaporins (AQPs), within the neurovascular unit has led to intensive research in understanding the underlying roles of each of the AQPs under normal conditions and in different diseases. In this review, we summarize some of the recent knowledge on AQPs, focusing on AQP4, the most abundant AQP in the central nervous system. Several experimental models illustrate that AQPs have dual, complex regulatory roles in edema formation and resolution. To date, no specific therapeutic agents have been developed to inhibit water flux through these channels. However, experimental results strongly suggest that this is an important area for future investigation. In fact, early inhibition of water channels may have positive effects in the prevention of edema formation. At later time points during the course of disease, AQP is important for the clearance of water from the brain into blood vessels. Thus, AQPs, and in particu-
\end{abstract}

lar AQP4, have important roles in the resolution of edema after brain injury. The function of these water channel proteins makes them an excellent therapeutic target.

Copyright $\odot 2011$ S. Karger AG, Basel

Brain edema is often manifested after the onset of cerebrovascular disease and significantly affects longterm outcome [1]. Noninvasive magnetic resonance imaging of the brain provides the ability to evaluate edema evolution after disease/injury onset, resolution, or after therapeutic interventions. The discovery of specific brain water channel proteins (aquaporins, AQPs) suggests new approaches to more accurately investigate water movement within the central nervous system. This review will acquaint the reader with the current state of brain AQP research, particularly after stroke. Finally, the future development of novel pharmacological tools targeted to AQPs to treat edema formation is also addressed.

\section{AQPs: Subgroups}

The AQP protein family exhibits a common structure with 6 membrane-spanning alpha-helical domains with intracellular carboxyl and amino termini and with an ap-

\section{KARGER}

(C) 2011 S. Karger AG, Basel

Fax +4161306 1234

E-Mail karger@karger.ch

www.karger.com
Accessible online at:

www.karger.com/ced
Jérôme Badaut, $\mathrm{PhD}$

Department of Pediatrics

Loma Linda University School of Medicine

Loma Linda, CA 92354 (USA)

Tel. +1 909558 8242,E-Mail jbadaut@llu.edu 
proximate molecular weight of $30 \mathrm{kDa}$. A common characteristic is a consensus motif Asn-Pro-Ala (NPA) that is implicated in pore formation [2]. The water channel family now consists of 13 members ubiquitously distributed throughout mammalian tissues that can be categorized into three subgroups:

(1) AQPs are composed of AQP0, 1, 2, 4, 5, 6 and 8, and represent the 'pure water channel family' primarily permeable only to water. However, there are exceptions, i.e. AQP6 and AQP8, which belong to this subgroup based on their gene sequences but AQP6 is also permeable to anions and AQP8 to urea [2].

(2) Aquaglyceroporins are composed of AQP3, AQP7, AQP9 and AQP10, and contribute to water diffusion, but they are also permeable to glycerol, urea and some monocarboxylates which can facilitate lactate diffusion [2].

(3) Super-AQPs have recently been proposed to include AQP11 and 12, which are localized to the cytoplasm. Their roles have not yet been fully determined but are thought to involve regulation of intracellular water transport, organelle volume and intravesicular homeostasis [2]

The majority of AQPs are assembled in homotetramers (fig. 1) where each individual AQP represents a water channel [3]. The assemblage of 4 molecules of AQP forms a central pore, through which water, ions and gases flow, depending on the AQP subtype. For example, the central pore is permeable to $\mathrm{O}_{2}, \mathrm{CO}_{2}$ and possibly nitric oxide in the case of AQP1, 4 and $5[3,4]$.

\section{AQPs in Normal Brain Tissues}

Currently, 7 AQP subtypes (AQP1, AQP3, AQP4, AQP5, AQP8, AQP9 and AQP12) have been described in cells within the rodent brain. AQP4, the most studied of these brain water channels, was first discovered in brain astrocytes (fig. 2) [5]. Other AQPs have also been well described in vivo (AQP1 and AQP9).

\section{AQP1: Cerebrospinal Fluid Formation}

AQP1 is primarily expressed within epithelial cells of the choroid plexus in the rodent brain and has been proposed to play a role in cerebrospinal fluid formation [6]. Recently, AQP1 has been described in a subpopulation of astrocytes in the nonhuman primate, suggesting species differences [7]. More importantly, it may have an additional role in brain water homeostasis [7]. The dorsal horn of the spinal cord and the trigeminal sensory ganglia have been identified as new cellular locations for AQP1 with a possible role in nociception [8].

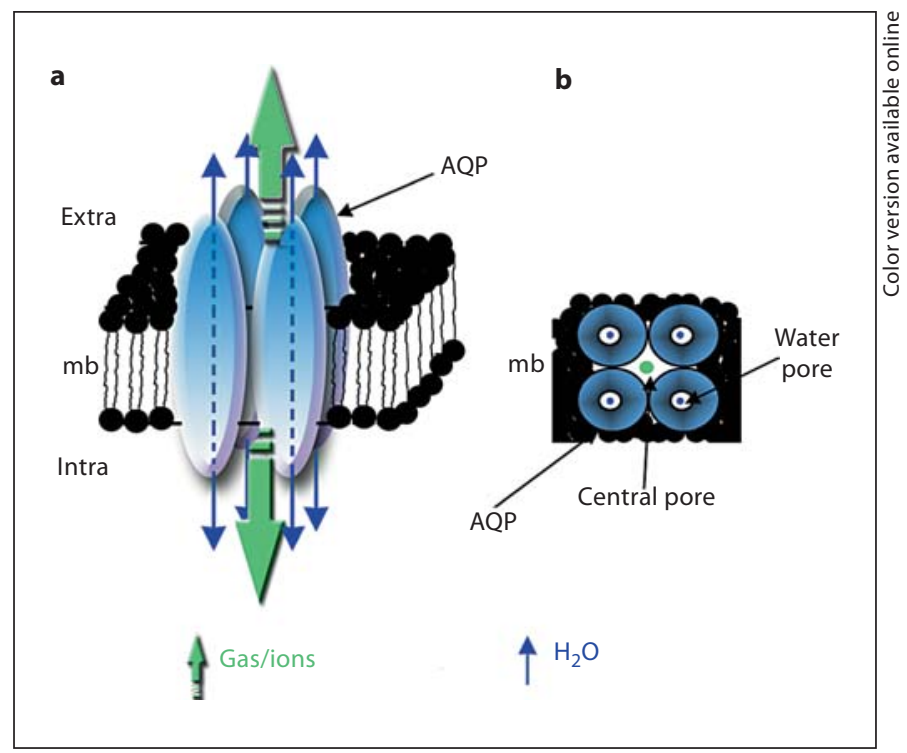

Fig. 1. Schematic of the AQP homotetramer assembly within the lipid membrane from a lateral (a) and a top view (b) resulting in a central pore permeable to cations and gases (thick arrows). Each individual AQP facilitates bidirectional water movement depending on the osmotic gradient (blue arrows, thin grey in the printed version). $\mathrm{mb}=$ Membrane.

\section{AQP4: Water Mobility in the Brain}

AQP4 is the most abundant water channel within the brain, in part due to its high concentration on astrocytic endfeet which are in contact with all cerebral blood vessels (fig. 2) [9, 10]. Further, colocalization of AQP4 and $\mathrm{K}_{\text {ir }} 4.1$ suggests that AQP4 may have a role in potassium homeostasis by facilitating water diffusion along the potassium gradient, and AQP4 knockout (KO) mice display a delay in potassium reuptake during electrical activity [11].

Electron-microscopic observations have demonstrated that the highest density of astrocyte endfoot AQP4 was observed in orthogonal arrays of proteins (OAPs) [12]. The organization of OAPs is based on two isoforms of AQP4: long (AQP4-m1) and short splice variants (AQP4$\mathrm{m} 23$ ) (fig. 3). The ratio of AQP4-m1 to AQP4-m23 determines the size of these OAPs [12], as the AQP4-m23 isoform stabilizes the OAP structure $[12,13]$ (fig. 3a). However, the exact functional roles of the OAPs remain unknown. An increase in the AQPm1 variant, however, disrupts the structure of the OAPs [12, 13] (fig. 3b).

AQP4 is known to be involved in water movement in vitro and in vivo in normal brain tissue [14], which underscores its importance in edema formation. Most studies have focused on the cerebral cortex where AQP4 is 


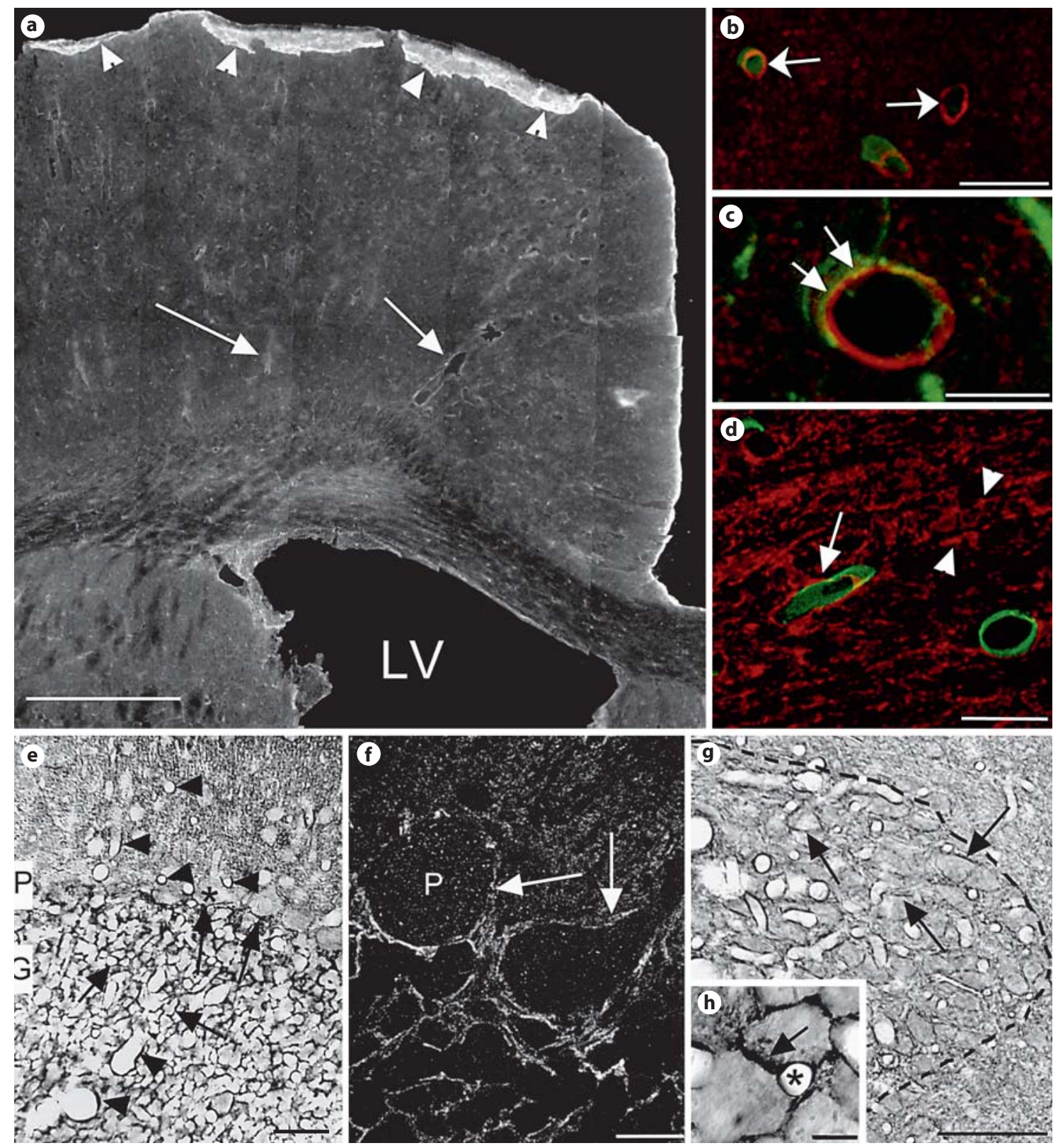

Fig. 2. AQP4 distribution within the rodent brain. a AQP4 is observed along intraparenchymal blood vessels (arrows) in the gray matter, ventricles, in the glia limitans (arrowheads) and ependymal cells along the lateral ventricle (LV). b AQP4-immunolabelling (red, arrows) is observed adjacent to the blood vessels but not on endothelial cells, stained by endothelial brain antigen (green). c AQP4-immunolabelling (red, arrows) on the astrocytic endfeet is revealed by anti-glial fibrillary acidic protein staining (green, arrows) within the parietal cortex. d In the corpus callosum, AQP4 expression (red) on astrocyte endfeet adjacent to blood vessels (endothelial brain antigen, green, arrows), cell bodies and processes (arrowheads). e AQP4 distribution in the cerebellum differs from the cortex. AQP4 is observed around the blood vessels (arrowheads) and also around the Purkinje cell bodies $\left(\mathrm{P},{ }^{*}\right)$ and neurons of the granular layer ( $G$, arrows). $f$ Higher magnification shows AQP4 expression at the basal membrane (arrows) of the cell bodies of Purkinje neurons (P) in the cerebellum. g, h A similar distribution was observed in the magnocellular hypothalamic nuclei such as in the paraventricular nucleus (dotted line). AQP4 is present on the astrocyte processes in contact with blood vessels ( ${ }^{*}$ in $\mathbf{h}$ ) and the magnocellular neurons in hypothalamic paraventricular nucleus (arrows). Bar: $\mathbf{a}=500 \mu \mathrm{m} ; \mathbf{b}, \mathbf{d}-\mathbf{f}=50 \mu \mathrm{m}$; $\mathbf{c}, \mathbf{h}=10 \mu \mathrm{m}$. 
Fig. 3. a Association between AQP4-m1 and AQP4-m23 isoforms contributes to form OAPs. Higher expression of AQP4$\mathrm{m} 23$ contributes to the formation of large OAPs. Recent knowledge on AQP led us to hypothesize that the large OAPs contribute to gas and cation diffusion in the astrocyte membranes through central pores (thick arrows). b Increased AQP4-m1, for example after stroke, induces disruption of OAPs (see [32]).

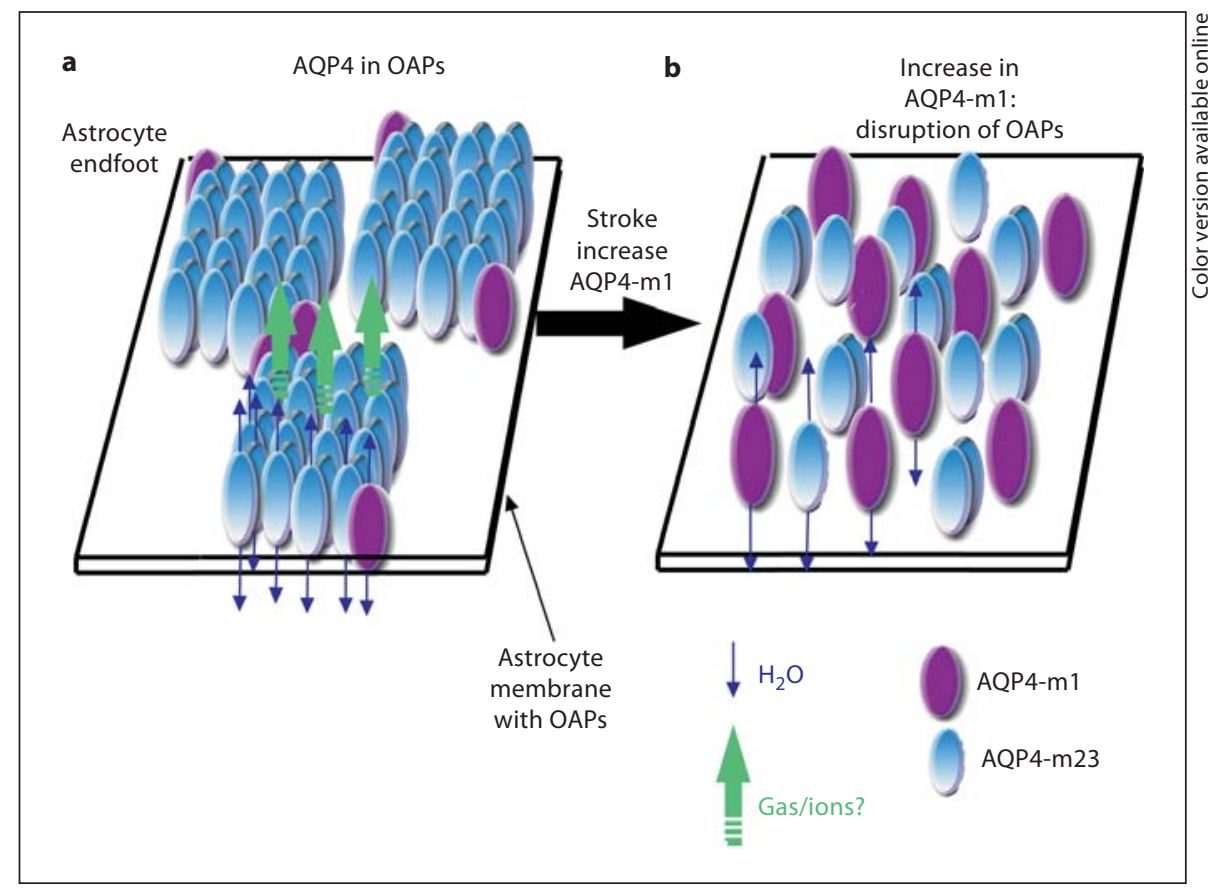

highly expressed on astrocytic endfeet in contact with blood vessels (fig. 2). However, astrocytic AQP4 distribution differs within brain structures, such as in the corpus callosum (fig. 2a, d), cerebellum (fig. 2e, f) and the magnocellular nuclei of the hypothalamus (fig. $2 \mathrm{~g}, \mathrm{~h}$ ) $[5,9]$. While AQP4 is primarily involved in water movement, recent data suggest that it may also contribute to cell adhesion [15], similar to the involvement of APQ0 in cell adhesion of epithelial cells in the lens [16]. These observations raise the question whether AQP4 is a structural molecule within the perivascular space. However, within the context of structural proteins, the role of AQP4 in the structure of the blood-brain barrier (BBB) remains to be resolved as there are conflicting reports $[17,18]$. Additional roles of AQP4 have also been suggested, including cell migration [19] and gas diffusion such as $\mathrm{CO}_{2}$ (fig. 1, 3).

\section{AQP9: Energy Metabolism}

AQP9 is an aquaglyceroporin that facilitates the diffusion of water and several solutes (e.g. glycerol, urea and monocarboxylate) and has been identified in rodent and primate brains [7]. Its highest level of expression is in the liver. Within the brain, it is found in three cell types: glia $[20,21]$, endothelial cells of subpial vessels [21] and neurons $[21,22]$. AQP9 expression in neuronal cells is found primarily in catecholaminergic neurons $[7,21,22]$. Two
AQP9 isoforms have been described, i.e. a short isoform $(26 \mathrm{kDa})$ found on the inner membrane of mitochondria and a long isoform $(30 \mathrm{kDa})$ located within the cell membrane [23].

Regulation of brain AQP9 shares similarities to the regulation AQP9 in hepatocytes, where expression is regulated by the blood insulin concentrations. AQP9 may play a key role in cellular energy balance as a glycerol channel in the liver and in the brain [24]. AQP9 is increased in catecholaminergic nuclei in diabetic rats [24], further suggesting that it likely participates in neuronal energy balance. In astrocytes, AQP9 can facilitate the diffusion of glycerol and monocarboxylates, which may serve as energy substrates in the central nervous system [21].

\section{Cerebrovascular Disease and Other Acute Injuries}

AQP1, AQP4 and AQP9 appear to play critical roles in edema formation and resolution in different forms of cerebrovascular disease and acute brain injuries, and are reviewed in the following sections.

AQP1 expression has been reported to be increased in human astrocytes after subarachnoid hemorrhage [25]. In rodent intracerebral hemorrhage models, induction of AQP1 expression has been demonstrated in cortical en- 

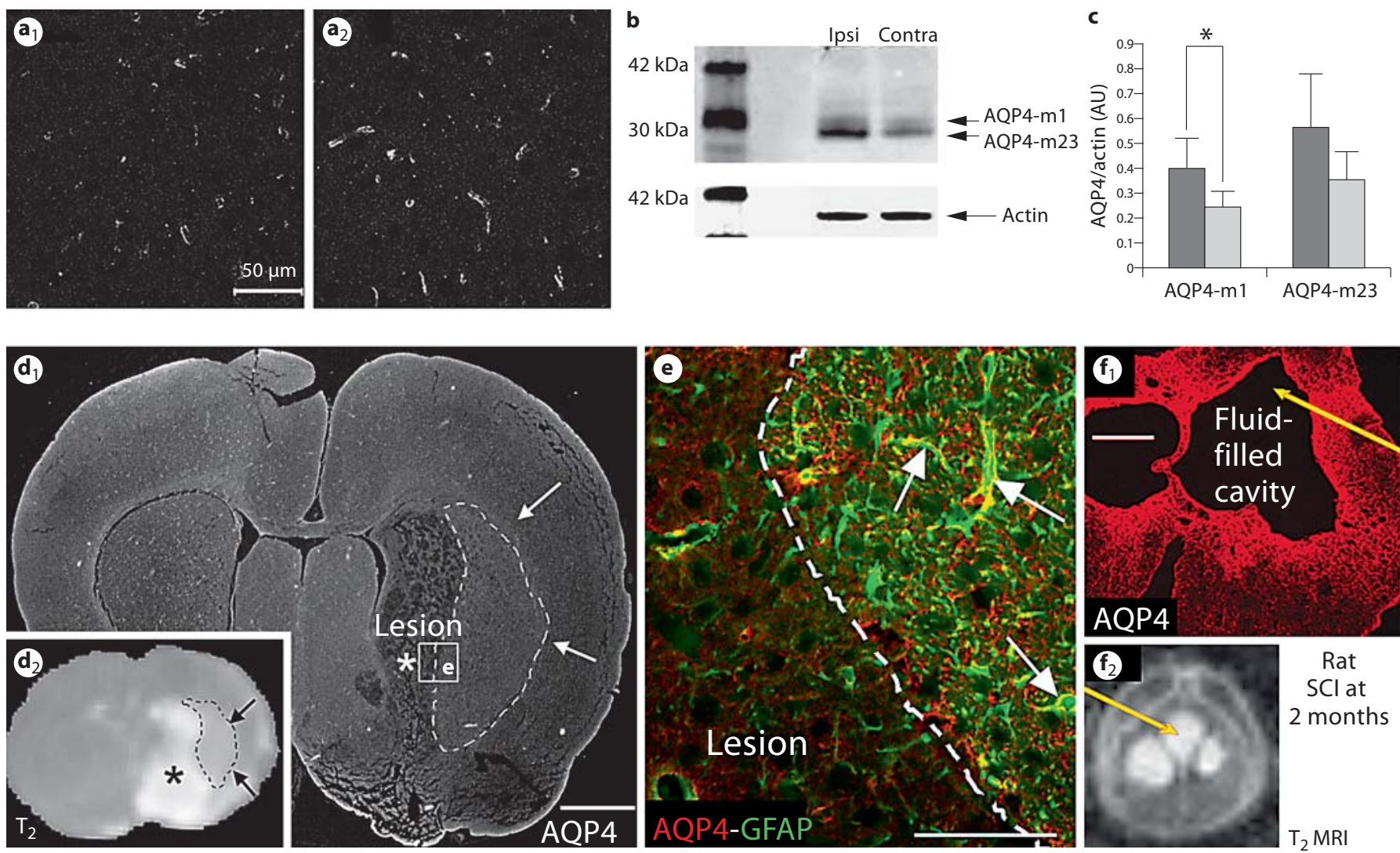

Fig. 4. AQP4s in cerebrovascular disease and injury. $\mathbf{a}_{1}, \mathbf{a}_{2} \mathrm{AQP} 4$ expression is increased in the striatum within the future lesion site $30 \mathrm{~min}$ after reperfusion in a model of MCA occlusion [22, 32, 35]. $\mathbf{a}_{1}$ Contralateral striatum. $\mathbf{a}_{2}$ Ipsilateral striatum. b AQP4 Western blots confirmed the increase in AQP4 at 30 min after reperfusion in the ispilateral striatum $[22,32,35]$. c AQP4 expression of isosforms $(n=7)$. Quantification showed an increase in the AQP4-m1 in the ipsilateral (dark grey column) striatum that validates the disruption of the OAPs observed after stroke [22, 32, 35]. Light grey column $=$ Contralateral striatum. $\mathbf{d}_{1}, \mathbf{d}_{2}$ AQP4 expression is decreased where $\mathrm{T}_{2} \mathrm{WI}$ images $\left(\mathbf{d}_{1}\right)$ showed hyperin- tense signals suggesting high water content $\left(^{*}\right)$ at day 28 after stroke. However, at the border of the lesion (dotted line) high expression of AQP4 correlated with differences in $\mathrm{T}_{2}$ signals (arrows). e Glial fibrillary acidic protein staining (green) revealed a glia scar adjacent to the hyperintense signal from the $\mathrm{T}_{2}$ images $\left(\mathbf{d}_{1}\right)$. The glia scar is characterized by a high level of AQP4 immunoreactivity (red, arrows). $\mathbf{f}_{1}, \mathbf{f}_{2}$ Similar observations were made in the spinal cord after injury in rats (SCI), where AQP4 expression was highly induced 2 months after a lesion. High expression of AQP4 observed around the cyst (yellow arrow) that was confirmed on $\mathrm{T}_{2} \mathrm{WI}\left(\mathbf{f}_{2}\right)$ [38]. Bar: $\mathbf{a}_{1}, \mathbf{e}=50 \mu \mathrm{m} ; \mathbf{d}_{1}=1 \mathrm{~mm} ; \mathbf{f}_{1}=200 \mu \mathrm{m}$. dothelial cells at $24 \mathrm{~h} \mathrm{[26],} \mathrm{further} \mathrm{suggesting} \mathrm{a} \mathrm{role} \mathrm{in}$ water movement during edema formation. Rodent spinal cord injury results in long-term induction of AQP1 in astrocytes and neurons [27]. While AQP1 expression in astrocytes is thought to contribute to the secretion to cerebrospinal fluid during cyst formation [27], neuronal AQP1 expression was colocalized with GAP-43, suggesting a role in plasticity and neurorepair [27]. These results illustrate the complexity of the pathophysiologic roles of AQP1.

Acute injury to the brain, including trauma [28], ischemia $[22,29]$ and human subarachnoid hemorrhage alter AQP4 expression [25]. The role of AQP4 in edema forma- tion was first described in an AQP4-KO model after permanent stroke showing that the presence of AQP4 contributes to postischemic cytotoxic edema [30, 31]. In trauma, AQP4 expression was shown to be downregulated within $24 \mathrm{~h}$ after injury [28]. The temporal evolution of AQP4 expression differs between stroke and other injury models [22]. In contrast to trauma, AQP4 expression is rapidly upregulated in astrocyte endfeet in contact with blood vessels, peaking at $1 \mathrm{~h}$ after stroke onset in a model of transient occlusion of the middle cerebral artery (MCA, fig. 4a) [22, 32]. This increase in AQP4 was observed at the site of the future lesion [22,32]. Thus, early administration of drugs to inhibit AQP4 ex- 
pression/function may be clinically useful. The increase in AQP4 expression is temporally correlated with the degree of brain swelling $[22,32]$ and the development of ionic brain edema (for a definition, see 'Edema Formation in Cerebrovascular Diseases: Revised Concepts' below).

A caveat is that early increased AQP4 expression is not observed in models of more severe stroke, such as permanent occlusion of the distal branch of the MCA [33], prompting the hypothesis that under great tissue duress the brain may not be able to synthesize sufficient new AQP4 proteins. After reperfusion, the ratio of AQP4-m1 and AQP4-m23 is changed in the ischemic hemisphere, with higher induction of AQP4-m1 compared to AQP4m23 observed by Western blot (fig. 4b, c) [32], suggesting disorganization of the OAPs (fig. 3b). Our findings are consistent with previous rat stroke data showing early OAP disruption [34]. We have shown that preconditioning leads to early induction of AQP4 as a potential protective mechanism by facilitating water removal, leading to decreased edema [32]. Further, disruption of the OAPs, which leads to increased AQP4-m1 content (fig. 3), likely modifies central pore function by altering diffusion of gases or cations.

In a transient MCA occlusion model, a second peak of AQP4 expression has been observed in the penumbra at $48 \mathrm{~h}$ that correlated with brain swelling [22, 32], and was associated with AQP4 induction on astrocytic endfeet, processes and cell bodies [22]. In mild stroke (30 min of MCA occlusion), elevated AQP4 expression was not observed past 7 days [22], but in more severe models, AQP4 expression remained increased up to 28 days in the border region of the remaining injury site and correlated with edema resolution [35].

Much of the evidence for a role of AQP4 in removal of excess water from tissues is based on studies that have shown increased edema formation in AQP4-KO mice after tissue perturbations $[36,37]$. We also have observed an excellent spatial/temporal correlation between AQP4 induction and decreased water content in rats subjected to ischemia [35]. However, late induction of AQP4 may also be related to glial scar formation (fig. $4 \mathrm{~d}$, e). In fact, AQP4 is involved in astrocytic migration in glial scar formation by facilitating water entry necessary for filopodia formation [19]. The presence of reactive astrocytic AQP4 expression in glial scars was observed 2 months after spinal cord injury [38] with high expression around the resultant cyst (fig. 4f), indicating a possible role for AQP4. Further complicating the picture is that high expression of AQP4 may contribute to cell adhesion [15], suggesting that AQP4 facilitates cell adhesion between astrocytes to form a new barrier or glia limitans.

The pattern of AQP4 expression in vascular disease reveals that AQP4 is a critical component regulating water movement during edema formation and resolution. It is important to note that the role of AQP4, either beneficial or deleterious, is still unclear and debated.

AQP9 protein is upregulated in reactive astrocytes along the infarct border after ischemic injury, with expression increasing gradually over time. However, AQP9 upregulation does not correlate with the degree of brain swelling, in contrast to AQP4 [20,22]. This would suggest that AQP9 does not play a significant role in edema formation after transient cerebral ischemia. Species-dependent changes in AQP9 have also been reported, where the temporal expression in mice is increased 7 days after stroke onset [22], but in gerbils a differential AQP9 expression is found after occlusion within hippocampal pyramidal neurons in CA1, CA2, CA3 at $6 \mathrm{~h}$ after stroke onset [39]. As pyramidal neurons do not express this channel under normal physiological conditions, it is likely that metabolic stressors may induce expression of this 'energy' channel. The functional consequences of increased AQP9 in neurons and astrocytes have not yet been elucidated. Interestingly, AQP9 permeability to lactate increases 4 -fold when the $\mathrm{pH}$ decreases to 5.5. Thus, ischemic lactic acidosis could increase the permeability of AQP9 to lactate, thus enabling the uptake of excess lactate by astrocytes $[20,22]$. AQP9 could then facilitate lactate movement between astrocytes and neurons, for use as an energy substrate, to assist in neuronal recovery $[20$, 22].

\section{Edema Formation in Cerebrovascular Diseases: Revised Concepts}

Cerebral edema after ischemia has been traditionally divided into two major classes: cytotoxic and vasogenic [40]. Cytotoxic edema is defined by intracellular water accumulation without $\mathrm{BBB}$ disruption while vasogenic edema appears after BBB disruption, leading to diffusion of proteins from the blood to the tissue followed by water accumulation in the extracellular space [40]. Recent advances in the molecular mechanisms of edema formation and $\mathrm{BBB}$ properties strongly suggest that these two categories represent a simplified view of more complex pathological processes. Simard et al. [41] have proposed adding a third subtype of edematous processes associated with cerebrovascular diseases, described as ionic edema. 


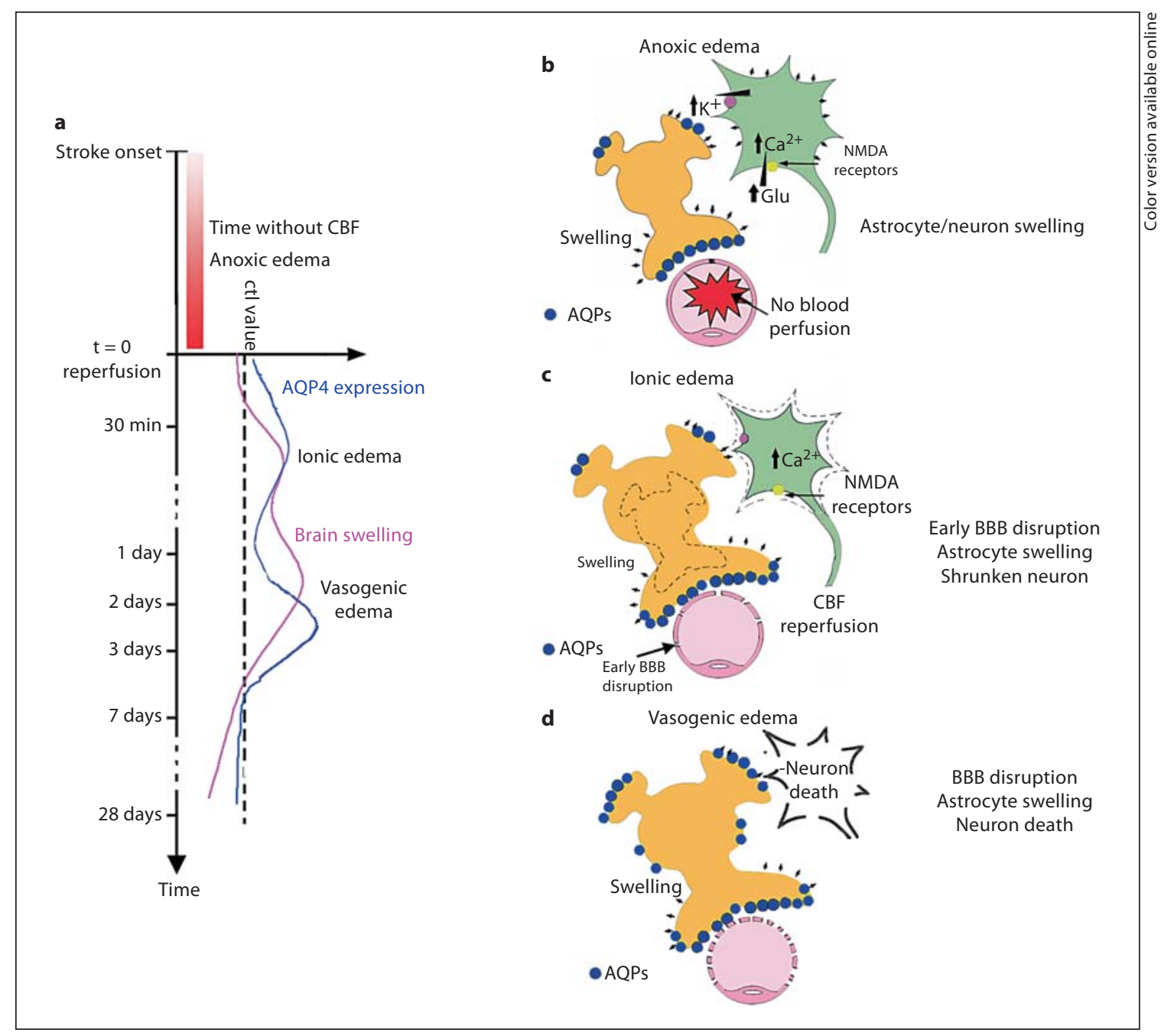

Fig. 5. Schematic drawing of the time course of edema formation after transient MCA occlusion (a) highlighting 3 different edema phases: anoxic (b), ionic (c) and vasogenic edema (d). a The first few minutes after an occlusion are characterized by anoxic edema (b) with loss of ionic gradients. The shear stress induced by reperfusion is followed by two peaks of brain swelling observed after transient MCA occlusion, one around 30 min coinciding with ionic edema (c) and a second at $48 \mathrm{~h}$ reflecting vasogenic edema (d). The other line indicates changes in the level of AQP4 expression following brain swelling (edema). $\mathbf{b}$ The initial anoxic edema is characterized as a swelling of the astrocytes and the neuronal

This is considered a transition form of edema from cytotoxic to vasogenic edema, where transcapillary sodium flux in endothelial cells occurs right before disruption of their tight junctions [41]. Based on this new subtype and the timeline of observed AQP changes in ischemia/stroke $[22,35]$, we suggest a further revision of the temporal mechanisms involved in edema formation after stroke dendrites caused by a disruption of the cellular ionic gradients and the entry of ions followed by water entry leading to cellular swelling through AQP4, transporters and ionic channels. c In ionic edema, astrocytes are still swollen and neuronal death starts, resulting in shrinkage of the neurons and shear stress on the nonperfused vascular tree that results in early transient leakage of the BBB. $\mathbf{d}$ Vasogenic edema is a result of disruption of the tight junctions between endothelial cells, leading to increased permeability of the cerebral blood vessels to albumin and other plasma proteins, further contributing to swelling of astrocytes and subsequent neuronal cell death.

(fig. 5a). We propose three major types: anoxic, ionic and vasogenic edema.

\section{Anoxic Edema}

The initial anoxic edema occurs within minutes after cessation of cerebral blood flow and is characterized as swelling of the astrocytes and neuronal dendrites (fig. 5b). 
Table 1. Summary of different treatments with a positive decrease on edema formation and correlation with AQP4 expression

\begin{tabular}{|c|c|c|c|c|}
\hline Treatment & Stroke model & $\begin{array}{l}\text { AQP4 expression } \\
\text { after ischemia } \\
\text { and treatment }\end{array}$ & $\begin{array}{l}\text { Time points } \\
\text { evaluated }\end{array}$ & Reference \\
\hline AQP4-KO & permanent MCAo in mouse & (no AQP4) & $24 \mathrm{~h}$ & Manley et al. [30] \\
\hline Edaravone & transient MCAo, $90 \mathrm{~min}$, rat & decreased & $24 \mathrm{~h}$ & Kikuchi et al. [58] \\
\hline Bumetanide & transient MCAo, $90 \mathrm{~min}$, mouse & decreased & $48 \mathrm{~h}$ & Migliati et al. [51] \\
\hline \multicolumn{5}{|l|}{ Phorbol 12-myristate } \\
\hline PKC activator & transient MCAo, 2 h, rat & decreased & $2 \mathrm{~h}$ & Kleindienst et al. [59] \\
\hline Propofol treatment & transient MCAo, $90 \mathrm{~min}$, rat & decreased & $24 \mathrm{~h}$ & Zheng et al. [60] \\
\hline $\mathrm{KO}$ mice $\mathrm{AR} / \mathrm{db}$ & transient MCAo, $30 \mathrm{~min}$ & decreased & $23.5 \mathrm{~h}$ & Yeung et al. [61] \\
\hline HS $10 \%$ & permanent MCAo in rat & decreased & $6,12,18 \mathrm{~h}$ & Zeng et al. [62] \\
\hline HS $7.5 \%$ & transient MCAo, $90 \mathrm{~min}$ & increased & $48 \mathrm{~h}$ & $\begin{array}{l}\text { Chen et al. [48]; } \\
\text { Zeylanov et al. [49] }\end{array}$ \\
\hline D-JNKI & $\mathrm{ICH}$ in mouse & increased & $6 \mathrm{~h}, 1,2$ or 5 days & Michel-Monigadon et al. [63] \\
\hline $\mathrm{V} 1$ antagonist & transient MCAo, $60 \mathrm{~min}$, mouse & increased & $24 \mathrm{~h}$ & Liu et al. [64] \\
\hline Preconditioning & transient MCAo, 30 min, mouse & increased & $1 \mathrm{~h}$ & Hirt et al. [32] \\
\hline Neuregulin & transient MCAo, $90 \mathrm{~min}$, rat & increased & $24 \mathrm{~h}$ & Li et al. [65] \\
\hline
\end{tabular}

MCAo = MCA occlusion; HS = hypertonic saline.

The cellular swelling within the first $10 \mathrm{~min}$ is a result of oxygen and glucose deprivation followed by a slow rise in extracellular $\left[\mathrm{K}^{+}\right][42]$. The absence of oxygen and energy nutrients induces a disruption of the cellular ionic gradients and leads to entry of ions into cells, accompanied by water entry in the cells which induces cellular swelling (fig. 5a, b). The potassium channel, $\mathrm{K}_{\mathrm{ir}} 4.1$, is often in close proximity to AQP channels and may facilitate water entry via AQP4 on astrocytic endfeet.

\section{Ionic Edema}

Anoxic edema may evolve to become ionic edema because the absence of oxygen and nutrients further alters the endothelial cell's ionic gradients, including transcapillary flux of $\mathrm{Na}^{+}[41,43]$ (fig. 5c). Moreover, reperfusion induces overpressure accompanied by shear stress on the nonperfused vascular tree that results in early transient leakage of the BBB $[22,32]$. This results in further entry of water through endothelial cells resulting in brain swelling within 30 min after reperfusion [22,32] and further increases BBB permeability [32, 44]. Astrocytes remain swollen and neuronal death starts, resulting in neuronal shrinkage (fig. 5c). While the exact role of AQP4 in this physiological cascade is still unclear, there is some evidence that decrements in water channel function may be beneficial [30]. Using syntrophin-KO mice without AQP4 on astrocytic endfeet appears beneficial by limit- ing the entry of water [45]. Conversely, in a preconditioning stroke model, higher induction of AQP4 was correlated with edema reduction [32].

\section{Vasogenic Edema}

The final step in this edema cascade is the development of vasogenic edema (fig. $5 \mathrm{~d}$ ), in which there is disruption of cerebrovascular endothelial tight junctions, leading to increased permeability to albumin and other plasma proteins [40]. Now a second peak of AQP4 expression is observed (fig. 5a). In AQP4-KO mice with induced vasogenic edema, the presence of AQP4 is required in assisting water clearance from tissues towards the blood and cerebrospinal fluid, the vasculature and ventricles [36].

\section{Water Movement and Neuroimaging}

Clinically, diffusion-weighted imaging and $\mathrm{T}_{2}$-weighted imaging $\left(\mathrm{T}_{2} \mathrm{WI}\right)$ are routinely used for diagnosis/prognosis in patients with cerebrovascular and other brain diseases. The quantitative diffusion-weighted imaging parameter, the apparent diffusion coefficient (ADC), is thought to reflect water mobility within brain tissues. Under pathological conditions, decreased ADC values are thought to reflect decreased extracellular space due to 
cellular swelling [46] (anoxic and ionic edema). The computed $\mathrm{T}_{2}$ value is representative of water content within brain tissues where increased $\mathrm{T}_{2}$ values correspond to late water accumulation (i.e. vasogenic edema) [46]. In stroke, ADC decreases within minutes and then $\mathrm{T}_{2}$ slowly increases within $24 \mathrm{~h}$ to days, by which time the ADC has normalized.

The magnitude of these magnetic resonance imaging changes has been correlated to patient outcomes [46], demonstrating that $\mathrm{ADC} / \mathrm{T}_{2}$ values are valuable biomarkers that can be used to monitor patients and treatment modalities. The cellular and molecular events underlying these multimodal imaging changes remain unclear [14]. At the present time, the early decrements in ADC are a combination of astrocytic and neuronal dendritic swelling after stroke onset [42] accompanied by decreased AQP4 expression [14, 29]. Recently, we demonstrated that ADC values can be decreased in normal animals using siRNA treatment against AQP4 with no change in $T_{2}$ [14]. Our results are in agreement with others, where early reduction in ADC values was correlated with a decrease in AQP4 staining after ischemia/hypoxia [29] and where increased ADC was correlated with AQP4 increases after hydrocephalus [47]. Together, these observations strengthen the contribution of the astrocytic network to changes in the expression of AQP4, as reflected by neuroimaging.

\section{Future Developments: Drugs against AQP4?}

AQP4 should be considered as a potential therapeutic target for reducing edema in patients with cerebrovascular disease or trauma [30, 36] (fig. 5). However, following treatment of stroke, heterogeneous alterations in AQP4 expression demonstrate the complexity of reducing edema (table 1). For example, hypertonic saline treatment induces increased or decreased AQP4 expression after stroke [48, 49]. It appears that the expression of AQP4 is dependent on the stroke model used, further complicating pharmacological manipulation to modulate this protein.

Currently, there are no specific inhibitors to block the AQP4 channel and such compounds are essential for evaluating the role and treatment of edema. Recent studies have proposed a range of compounds that may block AQP4, including bumetanide, which blocks the AQP4 channel and water permeability in oocytes [50]. However, bumetanide is also an inhibitor of $\mathrm{Na}-\mathrm{Cl}-\mathrm{K}$ cotransporter expressed in endothelial cells, complicating in vivo validation [43]. Bumetanide prevents edema formation in stroke models $[43,51]$, which correlates with decreased AQP4 expression [51], with the caveat that this efficacious action may be due to partial inhibition of the endothelial cell Na-Cl-K cotransporter.

Other investigators have proposed acetazolamide, a sulfonamide carbonic anhydrase inhibitor, as an inhibitor of water permeability associated with AQP1 and AQP4 $[52,53]$. However, it was reported that acetazolamide has no effect on water permeability $[54,55]$. Two other inhibitors belonging to the sulfonamide carbonic anhydrase inhibitor class - methazolamide and valproic acid - have also been tested but without clear benefits [53, 56]. Finally, erythropoietin has been shown to indirectly decrease the permeability of AQP4 via activation of metabotropic glutamate receptors [57]. Development of specific drugs targeting AQPs is required to further demonstrate the role of these channels in water modulation in cerebrovascular disease.

\section{Conclusions}

The cerebral vasculature and the BBB serve as important entry sites for large quantities of water when the BBB is disrupted. The neurovascular compartment consisting of blood vessels, neurons and astrocytes, is critical for the control of water mobility. The presence of AQP4 in astrocytes helps regulate brain water homeostasis. In this review, we have demonstrated the importance of AQPs in astrocytes and their putative role in edema formation. Development of new specific drugs to block water channels are clinically highly relevant and are also needed to provide a better understanding of the mechanisms involved in the regulatory roles of AQPs. This will aid development of appropriate pharmacological treatments of edema formation after brain injury and in patients with chronic diseases.

\section{Acknowledgments}

The authors thank Dr. O. Nesic for kindly providing figure 4c. Results illustrated in figure 2 are part of J.B.'s thesis from the UMR7519 in Strasbourg, France, under the supervision of Dr. F. Lasbennes.

This study was supported by the Swiss Science Foundation (FN 3100AO-108001, 31003A-122166 and IZK0Z3-128973) and supported in part by the NIH R01HD061946, Pediatric Research Fund, Department of Pediatrics and a NASA Cooperative Agreement NCC9-149 to the Department of Radiation Medicine at Loma Linda University. 


\section{References}

1 Klatzo I: Brain oedema following brain ischaemia and the influence of therapy. Br J Anaesth 1985;57:18-22.

2 Gonen T, Walz T: The structure of aquaporins. Q Rev Biophys 2006;39:361-396.

$\checkmark 3$ Yu J, Yool AJ, Schulten K, Tajkhorshid E: Mechanism of gating and ion conductivity of a possible tetrameric pore in aquaporin-1. Structure 2006;14:1411-1423.

$\checkmark 4$ Musa-Aziz R, Chen LM, Pelletier MF, Boron WF: Relative $\mathrm{CO}_{2} / \mathrm{NH}_{3}$ selectivities of AQP1, AQP4, AQP5, AmtB, and RhAG. Proc Natl Acad Sci USA 2009;106:5406-5411.

$\checkmark 5$ Nielsen S, Nagelhus EA, Amiry-Moghaddam M, Bourque C, Agre P, Ottersen OP: Specialized membrane domains for water transport in glial cells: high-resolution immunogold cytochemistry of aquaporin-4 in rat brain. J Neurosci 1997;17:171-180.

-6 Brown PD, Davies SL, Speake T, Millar ID: Molecular mechanisms of cerebrospinal fluid production. Neuroscience 2004;129:955968.

7 Arciénega II, Brunet JF, Bloch J, Badaut J: Cell locations for AQP1, AQP4 and 9 in the non-human primate brain. Neuroscience 2010;167:1103-1114.

$\checkmark 8$ Shields SD, Mazario J, Skinner K, Basbaum AI: Anatomical and functional analysis of aquaporin 1, a water channel in primary afferent neurons. Pain 2007;131:8-20.

-9 Badaut J, Nehlig A, Verbavatz J, Stoeckel M, Freund-Mercier MJ, Lasbennes F: Hypervascularization in the magnocellular nuclei of the rat hypothalamus: relationship with the distribution of aquaporin-4 and markers of energy metabolism. J Neuroendocrinol 2000;12:960-969.

-10 Badaut J, Verbavatz JM, Freund-Mercier MJ, Lasbennes F: Presence of aquaporin-4 and muscarinic receptors in astrocytes and ependymal cells in rat brain: a clue to a common function? Neurosci Lett 2000;292:75-78.

-11 Binder DK, Yao X, Zador Z, Sick TJ, Verkman AS, Manley GT: Increased seizure duration and slowed potassium kinetics in mice lacking aquaporin-4 water channels. Glia 2006;53:631-636.

-12 Rash JE, Davidson KGV, Yasumura T, Furman CS: Freeze-fracture and immunogold analysis of aquaporin-4 (AQP4) square arrays, with models of AQP4 lattice assembly. Neuroscience 2004;129:915-934.

- 13 Furman CS, Gorelick-Feldman DA, Davidson KG, Yasumura T, Neely JD, Agre P, Rash JE: Aquaporin-4 square array assembly: Opposing actions of $\mathrm{m} 1$ and $\mathrm{m} 23$ isoforms. Proc Natl Acad Sci USA 2003;100:13609-13614.

- 14 Badaut J, Ashwal S, Adami A, Tone B, Recker R, Spagnoli D, Ternon B, Obenaus A: Brain water mobility decreases after astrocytic aquaporin-4 inhibition using RNA interference. J Cereb Blood Flow Metab 2011;31:819831.
15 Hiroaki Y, Tani K, Kamegawa A, Gyobu N, Nishikawa K, Suzuki H, Walz T, Sasaki S, Mitsuoka K, Kimura K: Implications of the aquaporin-4 structure on array formation and cell adhesion. J Mol Biol 2006;355:628639.

16 Gonen T, Sliz P, Kistler J, Cheng Y, Walz T: Aquaporin-0 membrane junctions reveal the structure of a closed water pore. Nature 2004;429:193-197.

17 Saadoun S, Tait MJ, Reza A, Davies DC, Bell BA, Verkman AS, Papadopoulos MC: AQP4 gene deletion in mice does not alter bloodbrain barrier integrity or brain morphology. Neuroscience 2009;161:764-772.

18 Zhou J, Kong H, Hua X, Xiao M, Ding J, Hu $\mathrm{G}$ : Altered blood-brain barrier integrity in adult aquaporin-4 knockout mice. Neuroreport 2008;19:1-5.

19 Saadoun S, Papadopoulos MC, Watanabe H, Yan D, Manley GT, Verkman AS: Involvement of aquaporin-4 in astroglial cell migration and glial scar formation. J Cell Sci 2005; 118:5691-5698.

20 Badaut J, Hirt L, Granziera C, Bogousslavsky J, Magistretti PJ, Regli L: Astrocyte-specific expression of aquaporin-9 in mouse brain is increased after transient focal cerebral ischemia. J Cereb Blood Flow Metab 2001;21 477-482.

21 Badaut J, Petit JM, Brunet JF, Magistretti PJ, Charriaut-Marlangue C, Regli L: Distribution of aquaporin 9 in the adult rat brain: preferential expression in catecholaminergic neurons and in glial cells. Neuroscience 2004; $128: 27-38$

22 de Castro Ribeiro M, Hirt L, Bogousslavsky J, Regli L, Badaut J: Time course of aquaporin expression after transient focal cerebral ischemia in mice. J Neurosci Res 2006;83:12311240.

23 Amiry-Moghaddam M, Lindland H, Zelenin S, Roberg BA, Gundersen BB, Petersen P, Rinvik E, Torgner IA, Ottersen OP: Brain mitochondria contain aquaporin water channels: evidence for the expression of a short AQP9 isoform in the inner mitochondrial membrane. FASEB J 2005;19:14591467.

24 Badaut J, Brunet JF, Petit JM, Guerin CF, Magistretti PJ, Regli L: Induction of brain aquaporin 9 (AQP9) in catecholaminergic neurons in diabetic rats. Brain Res 2008; 1188:17-24.

25 Badaut J, Brunet JF, Grollimund L, Hamou MF, Magistretti PJ, Villemure JG, Regli L: Aquaporin 1 and aquaporin 4 expression in human brain after subarachnoid hemorrhage and in peritumoral tissue. Acta Neurochir 2003;86(suppl):495-498.

26 Yatsushige H, Ostrowski RP, Tsubokawa T, Colohan A, Zhang JH: Role of c-Jun N-terminal kinase in early brain injury after subarachnoid hemorrhage. J Neurosci Res 2007 85:1436-1448.
27 Nesic O, Lee J, Unabia GC, Johnson K, Ye Z, Vergara L, Hulsebosch CE, Perez-Polo JR: Aquaporin 1 - a novel player in spinal cord injury. J Neurochem 2008;105:628-640.

$>28$ Ke C, Poon WS, Ng HK, Pang JC, Chan Y: Heterogeneous responses of aquaporin-4 in oedema formation in a replicated severe traumatic brain injury model in rats. Neurosci Lett 2001;301:21-24.

$\checkmark 29$ Meng S, Qiao M, Lin L, Del Bigio MR, Tomanek B, Tuor UI: Correspondence of AQP4 expression and hypoxic-ischaemic brain oedema monitored by magnetic resonance imaging in the immature and juvenile rat. Eur J Neurosci 2004;19:2261-2269.

30 Manley GT, Fujimura M, Ma T, Noshita N, Filiz F, Bollen AW, Chan P, Verkman AS: Aquaporin-4 deletion in mice reduces brain edema after acute water intoxication and ischemic stroke. Nat Med 2000;6:159-163.

31 Zador Z, Stiver S, Wang V, Manley GT: Role of aquaporin-4 in cerebral edema and stroke. Handb Exp Pharmacol 2009:159-170.

\$2 Hirt L, Price M, Ternon B, Mastour N, Brunet JF, Badaut J: Early induction of AQP4 contributes the limitation of the edema formation in the brain ischemia. J Cereb Blood Flow Metab 2009;29:423-433.

$>33$ Friedman B, Schachtrup C, Tsai PS, Shih AY, Akassoglou K, Kleinfeld D, Lyden PD: Acute vascular disruption and aquaporin 4 loss after stroke. Stroke 2009;40:2182-2190.

>34 Suzuki M, Iwasaki Y, Yamamoto T, Konno H, Yoshimoto T, Suzuki J: Disintegration of orthogonal arrays in perivascular astrocytic processes as an early event in acute global ischemia. Brain Res 1984;300:141-145.

$\checkmark 35$ Badaut J, Ashwal S, Tone B, Regli L, Tian HR, Obenaus A: Temporal and regional evolution of aquaporin-4 expression and magnetic resonance imaging in a rat pup model of neonatal stroke. Pediatr Res 2007;62:248254.

-36 Papadopoulos MC, Manley GT, Krishna S, Verkman AS: Aquaporin-4 facilitates reabsorption of excess fluid in vasogenic brain edema. FASEB J 2004;18:1291-1293.

-37 Saadoun S, Papadopoulos MC: Aquaporin-4 in brain and spinal cord oedema. Neuroscience 2010;168:1036-1046.

38 Nesic O, Lee J, Ye Z, Unabia GC, Rafati D, Hulsebosch CE, Perez-Polo JR: Acute and chronic changes in aquaporin 4 expression after spinal cord injury. Neuroscience 2006; 143:779-792.

39 Hwang IK, Yoo KY, Li H, Lee BH, Suh HW, Kwon YG, Won MH: Aquaporin 9 changes in pyramidal cells before and is expressed in astrocytes after delayed neuronal death in the ischemic hippocampal CA1 region of the gerbil. J Neurosci Res 2007;85:2470-2479.

40 Unterberg AW, Stover J, Kress B, Kiening KL: Edema and brain trauma. Neuroscience 2004;129:1019-1027. 
41 Simard JM, Kent TA, Chen M, Tarasov KV, Gerzanich V: Brain oedema in focal ischaemia: Molecular pathophysiology and theoretical implications. Lancet Neurol 2007;6: 258-268.

42 Risher WC, Andrew RD, Kirov SA: Realtime passive volume responses of astrocytes to acute osmotic and ischemic stress in cortical slices and in vivo revealed by two-photon microscopy. Glia 2009;57:207-221.

-43 O’Donnell ME, Tran L, Lam TI, Liu XB, Anderson SE: Bumetanide inhibition of the blood-brain barrier $\mathrm{Na}-\mathrm{K}-\mathrm{Cl}$ cotransporter reduces edema formation in the rat middle cerebral artery occlusion model of stroke. J Cereb Blood Flow Metab 2004;24:10461056.

44 Strbian D, Durukan A, Pitkonen M, Marinkovic I, Tatlisumak E, Pedrono E, Abo-Ramadan U, Tatlisumak T: The bloodbrain barrier is continuously open for several weeks following transient focal cerebral ischemia. Neuroscience 2008;153:175-181.

45 Amiry-Moghaddam M, Otsuka T, Hurn PD, Traystman RJ, Haug FM, Froehner SC, Adams ME, Neely JD, Agre P, Ottersen OP, Bhardwaj A: An alpha-syntrophin-dependent pool of AQP4 in astroglial end-feet confers bidirectional water flow between blood and brain. Proc Natl Acad Sci USA 2003;100: 2106-2111.

46 Obenaus A, Ashwal S: Magnetic resonance imaging in cerebral ischemia: focus on neonates. Neuropharmacology 2008;55:271280.

-47 Tourdias T, Dragonu I, Fushimi Y, Deloire MS, Boiziau C, Brochet B, Moonen C, Petry KG, Dousset V: Aquaporin 4 correlates with apparent diffusion coefficient and hydrocephalus severity in the rat brain: a combined MRI-histological study. Neuroimage 2009;47:659-666.

48 Chen CH, Xue R, Zhang J, Li X, Mori S, Bhardwaj A: Effect of osmotherapy with hypertonic saline on regional cerebral edema following experimental stroke: a study utilizing magnetic resonance imaging. Neurocrit Care 2007;7:92-100.
49 Zeynalov E, Chen CH, Froehner SC, Adams ME, Ottersen OP, Amiry-Moghaddam M, Bhardwaj A: The perivascular pool of aquaporin- 4 mediates the effect of osmotherapy in postischemic cerebral edema. Crit Care Med 2008;36:2634-2640.

50 Migliati E, Meurice N, DuBois P, Fang JS, Somasekharan S, Beckett E, Flynn G, Yool AJ: Inhibition of aquaporin-1 and aquaporin-4 water permeability by a derivative of the loop diuretic bumetanide acting at an internal pore-occluding binding site. Mol Pharmacol 2009;76:105-112.

51 Migliati ER, Amiry-Moghaddam M, Froehner SC, Adams ME, Ottersen OP, Bhardwaj A: $\mathrm{Na}^{+}-\mathrm{K}^{+}-2 \mathrm{Cl}^{-}$cotransport inhibitor attenuates cerebral edema following experimental stroke via the perivascular pool of aquaporin-4. Neurocrit Care 2010;13:123-131.

52 Huber VJ, Tsujita M, Yamazaki M, Sakimura K, Nakada T: Identification of arylsulfonamides as aquaporin 4 inhibitors. Bioorg Med Chem Lett 2007;17:1270-1273.

53 Tanimura Y, Hiroaki Y, Fujiyoshi Y: Acetazolamide reversibly inhibits water conduction by aquaporin-4. J Struct Biol 2009;166: $16-21$.

54 Yang B, Zhang H, Verkman AS: Lack of aquaporin-4 water transport inhibition by antiepileptics and arylsulfonamides. Bioorg Med Chem 2008;16:7489-7493.

55 Sogaard R, Zeuthen T: Test of blockers of AQP1 water permeability by a high-resolution method: no effects of tetraethylammonium ions or acetazolamide. Pflügers Arch 2008;456:285-292.

56 Huber VJ, Tsujita M, Kwee IL, Nakada T: Inhibition of aquaporin 4 by antiepileptic drugs. Bioorg Med Chem 2009;17:418-424.

57 Gunnarson E, Song Y, Kowalewski JM, Brismar H, Brines M, Cerami A, Andersson U, Zelenina M, Aperia A: Erythropoietin modulation of astrocyte water permeability as a component of neuroprotection. Proc Natl Acad Sci USA 2009;106:1602-1607.
58 Kikuchi K, Tancharoen S, Matsuda F, Biswas KK, Ito T, Morimoto Y, Oyama Y, Takenouchi K, Miura N, Arimura N, Nawa Y, Meng X, Shrestha B, Arimura S, Iwata M, Mera K, Sameshima H, Ohno Y, Maenosono R, Tajima Y, Uchikado H, Kuramoto T, Nakayama K, Shigemori M, Yoshida Y, Hashiguchi T, Maruyama I, Kawahara K: Edaravone attenuates cerebral ischemic injury by suppressing aquaporin-4. Biochem Biophys Res Commun 2009;390:1121-1125.

-59 Kleindienst A, Fazzina G, Amorini AM, Dunbar JG, Glisson R, Marmarou A: Modulation of AQP4 expression by the protein kinase $\mathrm{C}$ activator, phorbol myristate acetate, decreases ischemia-induced brain edema. Acta Neurochir Suppl 2006;96:393-397.

60 Zheng YY, Lan YP, Tang HF, Zhu SM: Propofol pretreatment attenuates aquaporin-4 over-expression and alleviates cerebral edema after transient focal brain ischemia reperfusion in rats. Anesth Analg 2008;107: 2009-2016.

61 Yeung CM, Lo AC, Cheung AK, Chung SS, Wong D, Chung SK: More severe type 2 diabetes-associated ischemic stroke injury is alleviated in aldose reductase-deficient mice. J Neurosci Res 2010;88:2026-2034.

62 Zeng HK, Wang QS, Deng YY, Fang M, Chen $\mathrm{CB}, \mathrm{Fu} \mathrm{YH}$, Jiang WQ, Jiang X: Hypertonic saline ameliorates cerebral edema through downregulation of aquaporin-4 expression in the astrocytes. Neuroscience 2010;166: 878-885.

63 Michel-Monigadon D, Bonny C, Hirt L: cJun $\mathrm{N}$-terminal kinase pathway inhibition in intracerebral hemorrhage. Cerebrovasc Dis 2010;29:564-570

64 Liu X, Nakayama S, Amiry-Moghaddam M, Ottersen OP, Bhardwaj A: Arginine-vasopressin v1 but not v2 receptor antagonism modulates infarct volume, brain water content, and aquaporin-4 expression following experimental stroke. Neurocrit Care 2010; $12: 124-131$

65 Li Q, Li Z, Mei Y, Guo Y: Neuregulin attenuated cerebral ischemia-creperfusion injury via inhibiting apoptosis and upregulating aquaporin-4. Neurosci Lett 2008;443:155159. 